\title{
Oxidative coupling polymerization of octahydroxyquaternaphthyl derivatives producing stereocontrolled polybinaphthol
}

\author{
Shigeki Habaue (®), Kazutaka Ishikawa, Akiko Aikawa, Soichiro Murakami, \\ Bunpei Hatano
}

Department of Chemistry and Chemical Engineering, Faculty of Engineering, Yamagata

University, Yonezawa, Yamagata 992-8510, Japan

E-mail: habaue@yz.yamagata-u.ac.jp

Received: 11 January 2006 / Revised version: 10 April 2006 / Accepted: 10 April 2006 Published online: 25 April 2006 - @ Springer-Verlag 2006

\section{Summary}

The oxidative coupling polymerization of the novel quaternaphthyl monomers, such as $(S, S, S)-3,3$ '"-dihydroxy-2,2',3',2",3"2'"-hexamethoxy-1,1':4',1":4", 1'"'-quarternaphthyl and $(S, S, S)-3$ ',2"-dibenzyloxy-3,3'"-dihydroxy-2,2',3"2'"'-tetramethoxy$1,1 ': 4$ ',1":4",1'"'-quaternaphthyl, with the copper(I) chloride-(S)-2,2'-isopropyridenebis(4-phenyl-2-oxazoline) catalyst was performed. The obtained polybinaphthol derivatives consisted of about 20 repeating naphthalene units and the stereochemical composition of up to an $88 \%$ enantiomer excess $(S)$.

\section{Introduction}

1,1'-Bi-2-naphthol with an axial dissymmetry is one of the most important artificial chiral structures. The polymers bearing a binaphthol residue in the main- and sidechains have been studied. Especially, the poly(binaphthol)s, whose main-chain is comprised of continuously connected binaphthol units, are interesting from the viewpoints of their synthesis, conformation, physical and chiroptical properties, and functions. Two types of optically active poly(binaphthol)s are known (Scheme 1). One has a main-chain with a connection at the 6,6'-position of the binaphthol (type I) [1-6], and in the other, the binaphthol units are linked at their 4,4'-positions (type II) [7-14]. In the former, however, it was reported that the rotation at the single bond

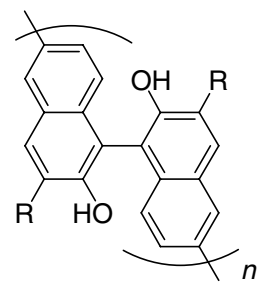

Type I

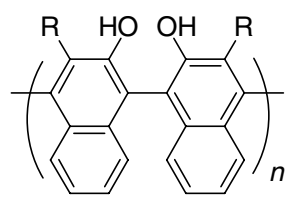

Type II

Scheme 1. 
between the 1,1'-bi-2-naphthol units cannot be restricted [3]. In contrast, the latter polymer with a continuous $R$ or $S$ stereostructure has a very stable one-handed helical main-chain conformation.

The type II oligomers with a stereochemically pure form have been synthesized by a stepwise approach [7-9]. On the other hand, the oxidative coupling polymerization (OCP) can also produce optically active poly(binaphthol)s, and we reported the asymmetric OCP of 2,3-dihydroxynaphthalene and 3,3'-dihydroxy-2,2'-dimethoxy1,1'-binaphthyl $\mathbf{1}$ with various chiral copper-diamine catalysts to afford a type II polymer with a controlled stereostructure to a certain extent (Scheme 2) [10-14].

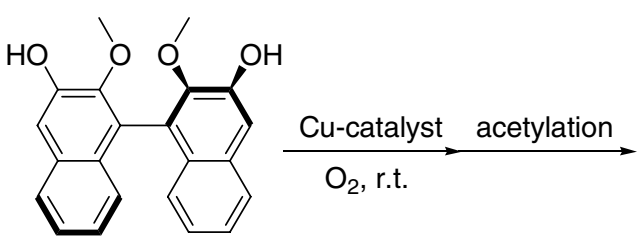

$(S)-1$

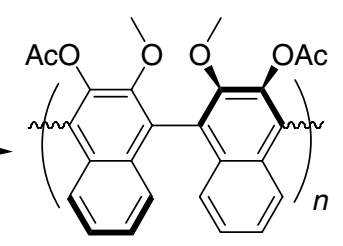

poly-(S)-1'

Scheme 2.

The asymmetric OCP of the designed monomer, i.e., optically pure oligonaphthyls, should be one of the most promising methods for the facile and highly stereocontrolled synthesis of the poly(binaphthol)s. The polymerization of $(S)$-1 with copper(I) chloride-(S)-2,2'-isopropyridenebis(4-phenyl-2-oxazoline) [(S)Phbox] [11] at room temperature under an $\mathrm{O}_{2}$ atmosphere proceeded with a diastereomer excess (d.e.) selectivity of $52 \%(\underline{S})$ to afford a polymer (poly- $(S)-\mathbf{1}$ '), that is, the enantiomer excess (e.e.) in the entire polymer chain was estimated to be $78 \%(S)$ from the d.e. value and number average molecular weight $\left(M_{\mathrm{n}}\right)$. The OCP of the ternaphthyl derivatives was also reported [12].

In this study, novel quaternaphthyl monomers, i.e., $(S, S, S)-3,3$ "'-dihydroxy2,2',3',2",3"2'"'-hexamethoxy-1,1':4',1":4", 1'"'-quaternaphthyl [(SSS)-2] and $(S, S, S)$ 3',2"-dibenzyloxy-3,3"'-dihydroxy-2,2',3"2'"'-tetramethoxy-1, 1':4', 1":4", 1'"'-quaternaphthyl [(SSS)-3] (Scheme 3), were synthesized and the OCP with copper catalysts, such as achiral di- $\mu$-hydroxo-bis $\left[\left(N, N, N^{\prime}, N^{\prime}\right.\right.$-tetramethylethylenediamine $)$ copper(II)] chloride [CuCl(OH)-TMEDA] [15-17] and chiral $\mathrm{CuCl}-(S)$ Phbox complexes, was carried out. To discriminate the stereochemistry between the monomer unit and the carbon-carbon bonds newly formed by the coupling reaction, the underlined absolute configurations, $\underline{R}$ and $\underline{S}$, are used for describing the latter stereostructure.

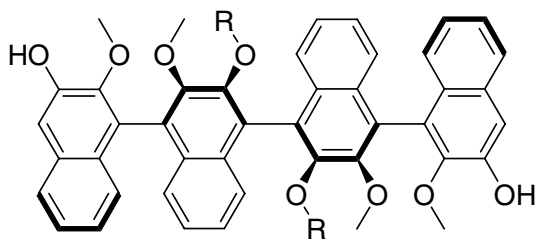

Scheme 3.

$\mathrm{R}=\mathrm{Me}:(S S S)-\mathbf{2} ; \mathrm{R}=\mathrm{Bn}:(S S S)-\mathbf{3}$ 


\section{Experimental}

\section{Measurements}

The ${ }^{1} \mathrm{H}$ and ${ }^{13} \mathrm{C}$ NMR spectra were measured using a Varian Unity-Inova spectrometer $\left(500 \mathrm{MHz}\right.$ for $\left.{ }^{1} \mathrm{H}\right)$ in $\mathrm{CDCl}_{3}$. The infrared (IR) spectra were recorded by a Horiba FT720 spectrometer. The mass (MS) spectra were obtained using a Jeol AX505H. The optical rotation was measured using a Jasco P-1010 polarimeter at $25{ }^{\circ} \mathrm{C}$. The circular dichroism (CD) spectra were recorded by a Jasco J-720L instrument. The size exclusion chromatography (SEC) analyses were performed by a Jasco PU-2080 equipped with a Jasco UV-2075 UV detector with KF-806F and KF-803F columns connected in series [eluent: tetrahydrofuran (THF), temp. $=25{ }^{\circ} \mathrm{C}$, flow rate $=1.0$ $\mathrm{ml} / \mathrm{min}]$. Calibration was carried out with standard polystyrenes.

\section{Materials}

The compound $(S)-\mathbf{1}$, reagents, and dry solvents used in the polymerization were synthesized or purchased as previously reported [10-12].

The monomers $(S S S)-\mathbf{2}$ and $(S S S)$-3 were synthesized by the coupling reaction of the mono-benzylated or mono-methoxymethylated $(S)-\mathbf{1}$ using $\mathrm{CuCl}(\mathrm{OH})$-TMEDA (20 mol\%) in $\mathrm{CH}_{2} \mathrm{Cl}_{2}$ at room temperature under an $\mathrm{O}_{2}$ atmosphere [8,12], followed by alkylation (methylation or benzylation) and deprotection of the terminal hydroxyl groups.

(SSS)-2: ${ }^{1} \mathrm{H}$ NMR (500 MHz, chloroform- $d$ ): $\delta 3.61\left(\mathrm{~s}, 6 \mathrm{H}, \mathrm{OCH}_{3}\right), 3.74(\mathrm{~s}, 6 \mathrm{H}$, $\left.\mathrm{OCH}_{3}\right), 3.79$ (s, 6H, $\left.\mathrm{OCH}_{3}\right), 6.19(\mathrm{~s}, 2 \mathrm{H}, \mathrm{OH}), 7.22-7.83(\mathrm{~m}, 18 \mathrm{H}$, aromatic). IR ( $\mathrm{KBr}$, $\mathrm{cm}^{-1}$ ): 3406 (br), 3060, 2935, 1506, 1466, 1450, 1387, 1346, 1240, 1111, 1014, 746. MS $(\mathrm{FAB}) m / z 719\left([\mathrm{M}+\mathrm{H}]^{+}\right) .[\alpha]_{\mathrm{D}}=-145(c=0.10, \mathrm{THF})$.

$(S S S)-3:{ }^{1} \mathrm{H}$ NMR $(500 \mathrm{MHz}$, chloroform- $d): \delta 3.64\left(\mathrm{~s}, 6 \mathrm{H}, \mathrm{OCH}_{3}\right), 3.66(\mathrm{~s}, 6 \mathrm{H}$, $\left.\mathrm{OCH}_{3}\right), 4.80\left(\mathrm{~d}, J=10.5 \mathrm{~Hz}, 2 \mathrm{H}, \mathrm{OCH}_{2^{-}}\right), 5.24\left(\mathrm{~d}, J=10.5 \mathrm{~Hz}, 2 \mathrm{H}, \mathrm{OCH}_{2^{-}}\right), 6.24(\mathrm{~s}$, $2 \mathrm{H}, \mathrm{OH}), 6.92-7.85$ (m, 28H, aromatic). IR (KBr, cm $\left.{ }^{-1}\right): 3446$ (br), 3062, 2938, 1504, $1467,1450,1396,1346,1242,1113,1007,746 . \mathrm{MS}(\mathrm{FAB}) m / z 870\left([\mathrm{M}]^{+}\right) .[\alpha]_{\mathrm{D}}=$ $-143(c=0.33, \mathrm{THF})$.

The model compound (SSS)-4' was also prepared by the acetylation of the coupling product of mono-benzylated $(S)-\mathbf{1}[(S S S)-4]$ [8].

(SSS)-4': ${ }^{1} \mathrm{H}$ NMR (500 MHz, chloroform- $d$ ): $\delta 2.04\left(\mathrm{~s}, 6 \mathrm{H}, \mathrm{CCH}_{3}\right), 3.50(\mathrm{~s}, 6 \mathrm{H}$, $\left.\mathrm{OCH}_{3}\right), 3.83\left(\mathrm{~s}, 6 \mathrm{H}, \mathrm{OCH}_{3}\right), 5.37\left(\mathrm{~s}, 4 \mathrm{H}, \mathrm{OCH}_{2}\right), 7.20-7.84(\mathrm{~m}, 28 \mathrm{H}$, aromatic). IR

Table 1. OCP of racemic monomers with copper catalysts

$([\mathrm{Cu}] /[$ monomer $]=0.2$, [monomer $]=0.35 \mathrm{M}$ (runs 1 and 2 ) or $0.17 \mathrm{M}$ (runs 3 and 4$)$, solvent = $\mathrm{CH}_{2} \mathrm{Cl}_{2}$ (runs 1 and 3) or THF (runs 2 and 4), temp. = room temperature, time $=48 \mathrm{~h}, \mathrm{O}_{2}$ atmosphere)

\begin{tabular}{ccccccc}
\hline Run & Monmer & Catalyst & $\begin{array}{c}\text { Yield }^{\mathrm{a}} \\
(\%)\end{array}$ & $\begin{array}{c}M_{\mathrm{n}} \times 10^{-3} \\
\left(M_{\mathrm{w}} / M_{\mathrm{n}}\right)^{\mathrm{b}}\end{array}$ & {$[\alpha]_{\mathrm{D}}{ }^{\mathrm{c}}$} & {$[\phi]_{\mathrm{D}}{ }^{\mathrm{c}}$} \\
\hline 1 & $(R / S)-\mathbf{1}$ & $\mathrm{CuCl}(\mathrm{OH})-$ TMEDA & 77 & $2.6(5.2)$ & --- & --- \\
2 & $(R / S)-\mathbf{1}$ & $\mathrm{CuCl}(S)$ Phbox & 84 & $3.3(1.6)$ & -70 & -300 \\
3 & $(R R R / S S S)-\mathbf{3}$ & $\mathrm{CuCl}(\mathrm{OH})-$ TMEDA & 85 & $5.1(3.1)$ & --- & --- \\
4 & $(R R R / S S S)-\mathbf{3}$ & $\mathrm{CuCl}-(R)$ Phbox & 62 & $4.0(2.0)$ & +36 & +343 \\
\hline
\end{tabular}

${ }^{\mathrm{a}} \mathrm{MeOH}$-insoluble part of the acetylated polymer; ${ }^{\mathrm{b}}$ determined by SEC in THF (polystyrene standard); 'in $\mathrm{CHCl}_{3}$ 
$\left(\mathrm{KBr}, \mathrm{cm}^{-1}\right): 2935,1770,1597,1465,1392,1255,1188,1113,1014,744 . \mathrm{MS}$ (FAB) $m / z 956\left([\mathrm{M}+\mathrm{H}]^{+}\right) \cdot[\alpha]_{\mathrm{D}}=-105(c=0.10, \mathrm{THF})$.

\section{Polymerization}

A monomer was added to a mixture of $\mathrm{CuCl}$ and bisoxazoline $([\mathrm{Cu}] /[$ monomer $]=$ 0.20 , $[$ monomer] $=0.17 \mathrm{M}$ ) in THF. After stirring the mixture at room temperature for $48 \mathrm{~h}$, the solvent was evaporated and acetylation was carried out using an excess amount of acetyl chloride and pyridine in $\mathrm{CH}_{2} \mathrm{Cl}_{2}$. The product was isolated as the methanol-insoluble part by centrifugation and drying in vacuo at $60{ }^{\circ} \mathrm{C}$ (Scheme 4).

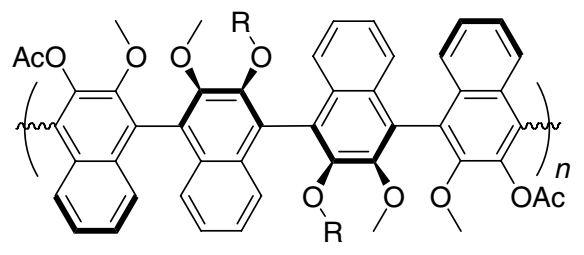

Scheme 4.

$$
\mathrm{R}=\text { Me: poly-(SSS)-2'; R = Bn: poly-(SSS)-3' }
$$

\section{Results and discussion}

Table 1 lists the results of the polymerization of the racemic monomers, such as $(R / S)$ 1 and $(R R R / S S S)-\mathbf{3}$, with copper catalysts at room temperature for $48 \mathrm{~h}$. In every run, the methanol-insoluble polymer was obtained in good yield. The polymers prepared with the chiral Phbox catalyst showed the absolute value of the molar rotation $\left([\phi]_{\mathrm{D}}\right)$ of 300 or greater (runs 2 and 4 ).

The CD spectra of poly- $(R / S)-\mathbf{1}$ ' and poly-(RRR/SSS $)-3$ ' are shown in Figure 1 . It was reported that the polymerization of the optically active monomers with the Phbox

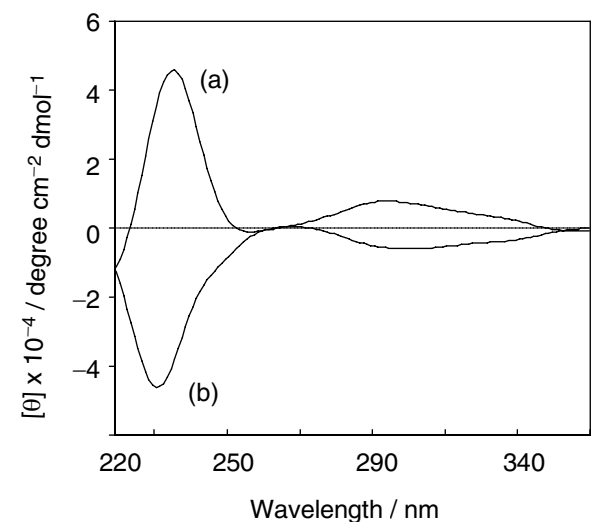

Figure 1. CD spectra of (a) poly-(R/S)-1' (Table 1, run 2) and (b) poly-(RRR/SSS)-3' (run 4) (binaphthalene unit, $\mathrm{CHCl}_{3}$ (a), THF (b)) 
Table 2. OCP of optically active monomers with copper catalysts

$([\mathrm{Cu}] /[$ monomer $]=0.2$, [monomer $]=0.35 \mathrm{M}$ (runs 1 and 2) or $0.17 \mathrm{M}$ (runs 3-6), solvent $=$ THF (runs 2, 4, and 6) or $\mathrm{CH}_{2} \mathrm{Cl}_{2}$ (runs 1, 3, and 5), temp. = room temperature, time $=24 \mathrm{~h}$ (runs 1 and 2) or $48 \mathrm{~h}$ (runs 3-6), $\mathrm{O}_{2}$ atmosphere)

\begin{tabular}{cccccccc}
\hline Run & Monmer & Catalyst & $\begin{array}{c}\text { Yield }^{\mathrm{a}} \\
(\%)\end{array}$ & $\begin{array}{r}M_{\mathrm{n}} \times 10^{-3} \\
\left(M_{\mathrm{w}} / M_{\mathrm{n}}\right)^{\mathrm{b}}\end{array}$ & $\begin{array}{c}\text { Stereoselectivity } \\
\text { d.e., }(\%)\end{array}$ & {$[\alpha]_{\mathrm{D}}{ }^{\mathrm{d}}$} & {$[\phi]_{\mathrm{D}}{ }^{\mathrm{d}}$} \\
\hline $1^{\mathrm{e}}$ & $(R)-1$ & $\mathrm{CuCl}(\mathrm{OH})-T M E D A$ & 71 & $5.2(1.6)$ & $22(\underline{R})(62(R))$ & +145 & +621 \\
$2^{\mathrm{e}}$ & $(S)-1$ & $\mathrm{CuCl}-(S)$ Phbox & 63 & $5.6(1.5)$ & $52(\underline{S})(78(S))$ & -137 & -587 \\
3 & $(S S S)-2$ & $\mathrm{CuCl}(\mathrm{OH})-\mathrm{TMEDA}$ & 69 & $4.0(2.2)$ & $2(\underline{S})(80(S))$ & -175 & -1401 \\
4 & $(S S S)-2$ & $\mathrm{CuCl}-(S)$ Phbox & 46 & $3.5(1.9)$ & $42(\underline{S})(88(S))$ & -188 & -1506 \\
5 & $(S S S)-3$ & $\mathrm{CuCl}(\mathrm{OH})-T M E D A$ & 80 & $5.3(2.7)$ & $14(\underline{S})(80(S))$ & -173 & -1649 \\
6 & $(S S S)-3$ & $\mathrm{CuCl}-(S)$ Phbox & 85 & $6.1(1.9)$ & $36(\underline{S})(86(S))$ & -178 & -1696 \\
\hline
\end{tabular}

${ }^{\mathrm{a}} \mathrm{MeOH}$-insoluble part of the acetylated polymer; ${ }^{\mathrm{b}}$ determined by SEC in THF (polystyrene standard); ${ }^{\mathrm{c}}$ determined by ${ }^{13} \mathrm{C}$ NMR analysis; in parentheses, the e.e. values for the stereochemical composition in whole polymer chain estimated from the $\underline{S}: \underline{R}$ and $M_{\mathrm{n}}$ values; din $\mathrm{CHCl}_{3}$; ${ }^{\mathrm{e}}$ reference 12

ligand proceeds under ligand stereocontrol, that is, the polymer with an $\underline{S}$ - or $\underline{R}-$ selectivity is produced by the $(S)$ - or $(R)$-Phbox regardless of the monomer chirality, as well as the monomer structure [12]. During the polymerization of the racemic monomer, the optically active polymer due to the progress of the enantiomer-selective reaction [14], in addition to the stereoselective bond formation, can be obtained. Although the details are not clear at present, the polymer preferentially having an $S$ - or $R$-configuration was obtained using the $(S)$ - or $(R)$-Phbox, respectively, based on the CD spectral patterns.

The OCP results of $(S S S)-\mathbf{2}$ and $(S S S)$-3 at room temperature under an $\mathrm{O}_{2}$ atmosphere are summarized in Table 2, together with those for the polymerization of $\mathbf{1}$ (runs 1 and 2) $[10,12]$. The obtained methanol-insoluble polymers from the quaternaphthyls showed an $M_{\mathrm{n}}$ value around $4-5 \times 10^{3}$, therefore, these are the oligomers with approximately 5-6 monomer units, which correspond to about 20 repeating units with respect to the naphthalene.

In previous studies, the stereochemistry of the bond newly formed during the polymerization $(\underline{R}: \underline{S})$ can be estimated from the methyl carbon absorption of the acetyl groups in the ${ }^{13} \mathrm{C}$ NMR spectrum for the polymer obtained from the optically active monomers [10,12]. Figure 2 shows the ${ }^{13} \mathrm{C}$ NMR spectrum of poly- $(S S S)-2$ ' obtained using the $\mathrm{CuCl}-(S) \mathrm{Phbox}$ catalyst (run 4$)$, and the peaks are assigned as

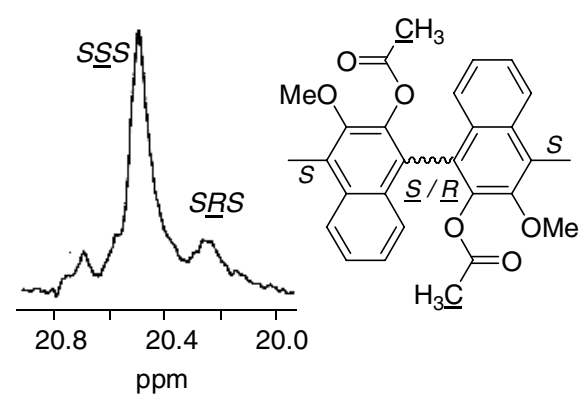

Figure 2. ${ }^{13} \mathrm{C}$ NMR spectrum of poly-(SSS)-2' obtained with (S)Phbox (Table 2, run 4) $\left(\mathrm{CDCl}_{3}\right.$, $\left.60{ }^{\circ} \mathrm{C}\right)$ 
shown in the figure, based on the fact that $(S S S)$-2 was used as a monomer. The evaluated stereoselectivity (d.e. value) is listed in Table 2, together with the e.e. value for the stereochemical composition in the whole polymer chain calculated from the d.e. and $M_{\mathrm{n}}$ values [10,12].

The monomer configuration affected the coupling stereoselectivity during the polymerization with the achiral $\mathrm{CuCl}(\mathrm{OH})$-TMEDA complex (runs 3 and 5), and the polymers showed an $S$ - and poor selectivity of 2 and 14\% d.e., which were lower than that observed for the poly- $(S)-\mathbf{1}$ ' obtained using the same catalyst system (run 1). However, their e.e. values were calculated to be $80 \%$ e.e. $(S)$ and significantly higher, indicating that the quaternaphthyl monomer structure is effective for the stereocontrolled synthesis of the type-II polybinaphthol. Although the coupling stereoselectivity observed for the polymerization of the quaternaphthyl monomers with the $(S)$ Phbox catalyst (runs 4 and 6) reduced in comparison to that observed for poly-(S)-1' (run 2), they again showed a higher stereochemical composition in the whole polymer chain of $86-88 \%$ e.e. $(S)$. The polymers obtained from $(S S S)-2$ and $(S S S)-3$ also had higher absolute values for the specific rotation $\left([\alpha]_{\mathrm{D}}\right)$ and $[\phi]_{\mathrm{D}}$ than those of poly-1'.

Figure 3 depicts the CD spectra of the obtained polymers together with that for the model compounds, $\quad(S, S, S)-3,3$ '"'-diacetoxy-2,2',3',2",3"'2'"-hexamethoxy-

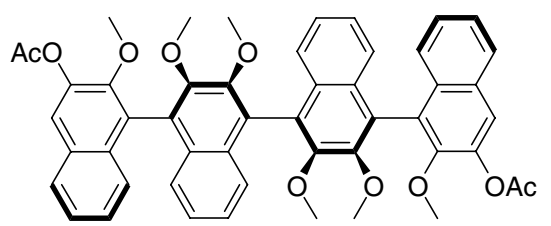

$(S S S)-2^{\prime}$

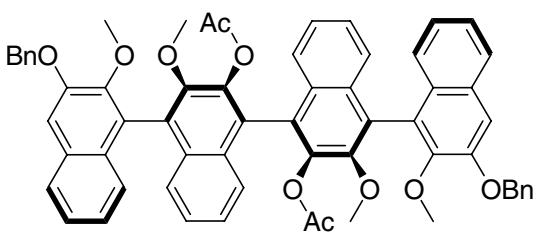

(SSS)-4'

Scheme 5 .
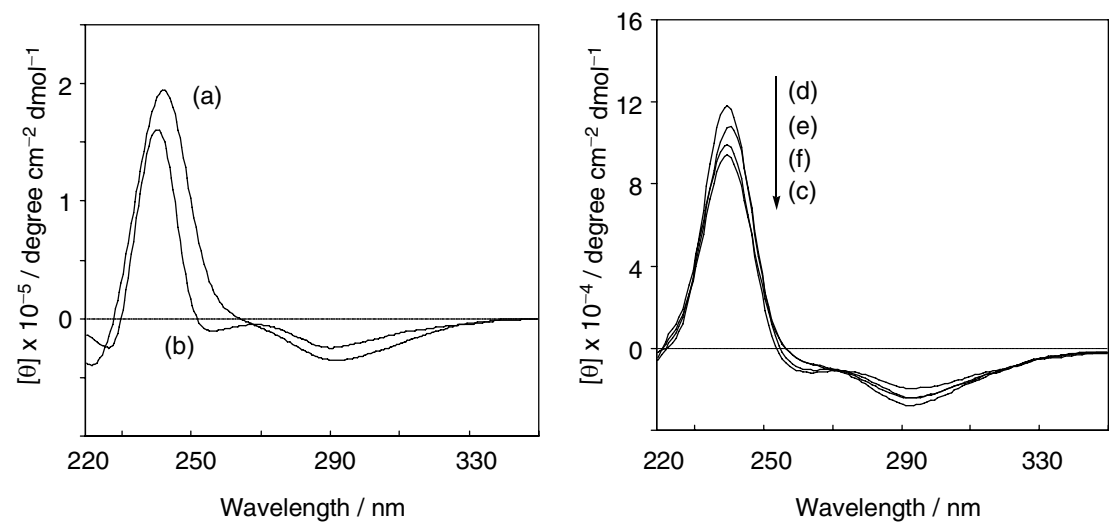

Figure 3. CD spectra of (a) (SSS)-2', (b) (SSS)-4', (c) poly-(SSS)-2' obtained with TMEDA (Table 2, run 3), (d) poly-(SSS)-2' obtained with (S)Phbox (run 4), (e) poly-(SSS)-3' obtained with TMEDA (run 5), and (f) poly-(SSS)-3' obtained with (S)Phbox (run 6) (binaphthalene unit, $\mathrm{CHCl}_{3}$ ) 
Table 3. Model oxidative coupling of $(S)$-Bn-1 with copper catalysts $\left([\mathrm{Cu}] /[\mathrm{Bn}-1]=0.2,[\mathrm{Bn}-1]=0.35 \mathrm{M}\right.$, solvent $=\mathrm{CH}_{2} \mathrm{Cl}_{2}$ (run 1) or THF (runs 2 and 3 ), temp. = room temperature, time $=2 \mathrm{~h}, \mathrm{O}_{2}$ atmosphere)

\begin{tabular}{cccc}
\hline Run & Catalyst & Yield $^{\mathrm{a}}(\%)$ & Selectivity $(S \underline{\underline{S}} S: S \underline{R} S)$ \\
\hline 1 & $\mathrm{CuCl}(\mathrm{OH})-\mathrm{TMEDA}^{\mathrm{b}}$ & 51 & $84: 16$ \\
2 & $\mathrm{CuCl}-(S)$ Phbox & 35 & $69: 31$ \\
3 & $\mathrm{CuCl}-(R)$ Phbox & 27 & $33: 67$ \\
\hline
\end{tabular}

${ }^{\mathrm{a}}$ isolated yield; ${ }^{\mathrm{b}}$ reaction time $=2.5 \mathrm{~h}$

1,1':4',1":4",1'"'-quaternaphthyl [(SSS)-2'] [7] and (SSS)-4' (Scheme 5). The spectral pattern of the polymers very closely resembles that of the model compounds, and the absorption intensity around $240 \mathrm{~nm}$ for the polymers was lower than that for the models.

The model oxidative coupling reaction using the mono-benzylated $(S)-\mathbf{1}[(S)$-Bn-1] as a substrate at room temperature for $2 \mathrm{~h}$ under an $\mathrm{O}_{2}$ atmosphere was carried out (Scheme 6) and the results are shown in Table 3. The $(S)$ - and $(R)$-Phbox catalysts showed a selectivity of $38 \%$ d.e. $(\underline{S})$ and $34 \%$ d.e. $(\underline{R})$, respectively (runs 2 and 3 ). These results are very comparable to the polymerization results and demonstrate that the coupling reaction proceeds in a ligand controlled manner. The reaction with the TMEDA catalyst afforded coupling products with a d.e. of $68 \%(S)$. Although the value is much higher than that observed for the polymerization, the reaction produces coupling products under substrate control, which is again in good agreement with the polymerization.

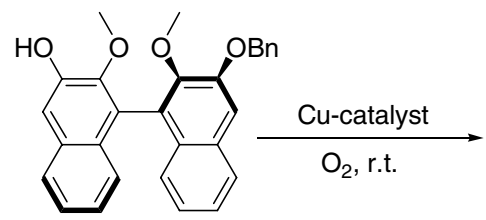

(S) $-\mathrm{Bn}-1$

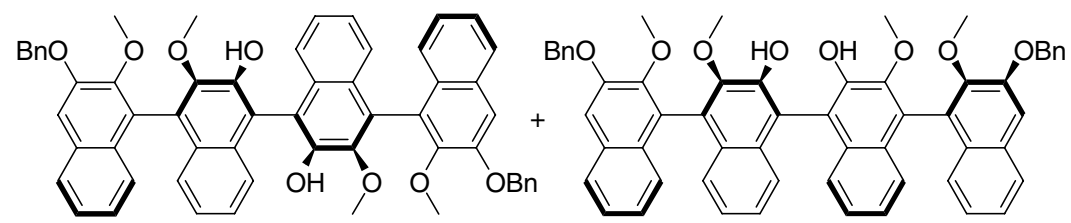

Scheme 6.

(SSS)-4

$(S R S)-4$

\section{Conclusions}

The oxidative coupling polymerization of the optically active quaternaphthyl derivatives with the chiral copper(I)-bisoxazoline $(\mathrm{CuCl}-(S) \mathrm{Phbox})$ catalyst produced a polybinaphthol derivatives with a stereochemistry of up to $88 \%$ e.e. The coupling reaction proceeded in a ligand controlled fashion, and this polymerization system should be valuable for producing a controlled polybinaphthol skeleton. 
Acknowledgements. This work was partially supported by SHISEIDO Grant for Scientific Research and Grant-in-Aid for Scientific Research (No. 15350066) from the Ministry of Education, Science, Sports, and Culture of Japan.

\section{References}

1. Hu Q-S, Zheng X-F, Pu L (1996) J Org Chem 61:5200

2. Hu Q-S, Vitharana D, Zheng X-F, Wu C, Kwan CMS, Pu L (1996) J Org Chem 61:8370

3. Wayatt SR, Hu Q-S, Yan X-L, Bare WD, Pu L (2001) Macromolecules 34:7983

4. Ma L, White PS, Lin W (2002) J Org Chem 67:7577

5. Xie X, Phuan P-W, Kozlowski MC (2003) Angew Chem Int Ed 42:2168

6. Temma T, Habaue S (2005) J Polym Sci A Polym Chem 43:6287

7. Fuji K, Furuta T, Tanaka K (2001) Org Lett 3:169

8. Tsubaki K, Miura M, Morikawa H, Tanaka H, Kawabata T, Furuta T, Tanaka K, Fuji K (2003) J Am Chem Soc 125:16200

9. Furuta T, Tanaka K, Tsubaki K, Fuji K Tetrahedron 60:4431

10. Habaue S, Seko T, Okamoto Y (2002) Macromolecules 35:2437

11. Habaue S, Seko T, Okamoto Y (2003) Macromolecules 36:2604

12. Habaue S, Seko T, Isonaga M, Ajiro T, Okamoto Y (2003) Polym J 35:592

13. Habaue S, Ajiro H, Yoshii Y, Hirasa T (2004) J Polym Sci A Polym Chem 42:4528

14. Habaue S, Ishikawa K (2005) Polym Bull 55:243

15. Nakajima M, Hashimoto S, Noji M, Koga K (1998) Chem Pharm Bull 46:1814

16. Sasada Y, Shibasaki Y, Suzuki M, Ueda M (2003) Polymer 44:355

17. Habaue S, Ohnuma M, Mizoe M, Temma T (2005) Polym J 37:625

18. Tanaka K, Furuta T, Fuji K, Miwa Y, Taga T (1996) Tetrahedron Asymmetry 7:2199 